ISSN 2504-1916 Vol. 16/1 (2017), 115-123

\title{
Jazz der 1960er-Jahre in der Schweiz
}

\section{Vorboten eines Aufbruchs}

Bruno Spoerri

Bruno Spoerri kennt die Geschichte des Schweizer Jazz wie kein zweiter: als junger Saxofonist nahm er 1954 mit dem Basler „New Cool Team" am Amateur Jazz Festival Zürich teil und gehörte damit zu den ersten Protagonisten des Cool Jazz in der Schweiz. Er war Mitglied in zablreichen Jazz-Ensembles, wie zum Beispiel dem „Francis Notz Septet“ (zusammen mit George Gruntz), dem „Metronome Quintet", der „Modern Jazz Group Freiburg“ und dem „Hans Kennel Quintet“. Er erlebte die Blütezeit des legendären Zürcher Jazzclubs Africana in den früben 1960er-Jahren hautnah mit und war um 1970 mit der Band "Jazz Rock Experience" einer der Jazzrock-Pioniere in der Schweiz: Gleichzeitig begann er, Elektronik mit Jazzimprovisation zu verbinden. Spoerris Beitrag zu dieser Sonderausgabe von EJM ist aus der Perspektive eines Zeitzengen verfasst, der den Jazz in der Schweiz seit den 1960er-Jahren mitgeprägt hat. (Hrsg.)

$\mathrm{E}$ s ist unbestritten: In den 1960er-Jahren veränderten sich das Bild ebenso wie die Bedeutung des Jazz wesentlich. Nicht nur des Jazz natürlich: Es kam die Zeit der Jugendrevolten, der Protestbewegungen in der ganzen Welt und bald auch die Zeit des technologischen Umbruchs. Der Jazz verlor seine Rolle als die Musik der jungen Generation, gleichzeitig aber entstanden Jazzschulen mit ihren weitreichenden Folgen.

Die Generation der um 1940 Geborenen hat diese Zeit wahrscheinlich am intensivsten erlebt; diese Generation war damals 20- bis 30-jährig, hatte ihre Adoleszenz in den 1950er-Jahren durchgestanden und hatte so auch die Vorboten des Aufbruchs erlebt.

Das Erlebnis einer Epoche ist etwas anderes als die nachträgliche Aufarbeitung, auch anhand der besten Quellen. Die Erinnerung ist oft unsystematisch und möglicherweise verklärt, aber gleichzeitig von einer Qualität, die später kaum mehr nachvollziehbar ist und allenfalls von einem begabten Romanautor vermittelt werden könnte. Ich möchte versuchen, eine Sicht von innen auf diese Zeit zu geben - nicht die Sicht des erklärenden Historikers oder des verstehenden Soziologen, sondern die des Zeitzeugen, der oft Wege ging, die ihn an andere Orte hinführten als erwartet.

„Aufbruch im Schweizer Jazz“ - das lässt vermuten, dass die Schweizer Jazzer eines Tages die Wanderschuhe hervornahmen und dann geradeaus in Richtung neuer Ziele aufbra- 
chen. Natürlich war es nicht so einfach: Geradlinigkeit in der Entwicklung des Jazz finden wir nur in populären Übersichtsdarstellungen, wie etwa im altehrwürdigen Jazzbuch von Joachim Ernst Berendt ${ }^{1}$ - die Wirklichkeit ist komplizierter, viel weniger zielgerichtet und meist auch widersprüchlich. Das trifft ganz besonders auf die 50er- und 60er-Jahre des 20. Jahrhunderts zu, eine Zeit, die vor allem für die damalige Jugend tiefgreifende Änderungen brachte.

Unsere Generation (geboren um 1930-40) wurde in eine Zeit der Enge und Unsicherheit hineingeboren und erlebte dann den wirtschaftlichen Aufstieg der 1950er-Jahre. Gerade weil die Verhältnisse so eng waren, war jede Öffnung ein zaghafter Akt, der von viel Unsicherheit begleitet war, es war ein Aufbruch mit Scheuklappen.

Je genauer ich diese Epoche anschaue, desto mehr Widersprüche entdecke ich, desto öfter tauchen ungelöste Fragen auf. Wir können diese Zeit nur verstehen, wenn wir zunächst genau hinschauen und erst dann verallgemeinern. Wir müssen über die beteiligten Musiker mehr wissen, das Repertoire der Orchester kennen, erforschen, wann welche Musik hier erstmals gehört werden konnte. Wer war Veranstalter, was gab es an Konzertorten, wie hoch waren die Gagen? Wie haben die Zeitzeugen diese Zeit subjektiv erlebt? Wie hat ihre Umwelt auf den Wandel der Musik reagiert? Fragen, die beantwortet werden müssen, bevor Folgerungen gezogen werden.

„History is not what happened. History is what it felt to be there when it happened.“ (George Kennan, Historiker und Diplomat, 1904-2001)²

To be there - nicht mehr viele sind übrig, die um 1960 dabei waren. Hier eine - sicher unvollständige - Liste: Franco Ambrosetti, Elsie und Siro Bianchi, Franz Biffiger, Victor Burghardt, Peter Candiotto, Hans Deyssenroth, Isla Eckinger, Pierre Favre, Peter Fürst, Karl Theodor „KT.“ Geier, Peter Giger, Martin Hugelshofer, Peter Jacques, Werner Keller, Beat Kennel, Hans Kennel, Klaus Koenig, Vali Mayer, Tutilo Odermatt, Peter Rüedi, Aldo Sandmeier, Godi Schaaf, Mario Schneeberger, Irène Schweizer, Willy Seefeldt, Siro Spörli, Arild Wideroe, Theo Zwicky.

Diese Personen müssten befragt werden.

Ich kann hier nur ein paar knappe Momentaufnahmen bieten, die zu zeigen versuchen, wie unsere Generation, die der kurz vor dem 2. Weltkrieg geborenen, funktionierte.

\section{Momentaufnahme 1: 1948, Basel}

Wir wuchsen auf in einer Welt der fast absoluten Autoritätsgläubigkeit, in der Lehrer Ohrfeigen austeilten, Jugendliche nicht wagten, im Tram Sitzplätze zu besetzen, in den Schulen absolute Geschlechtertrennung bestand und auch soziale Unterschiede eine große Rolle spielten - die

1 Joachim Ernst Berendt, Das Jazzbuch. Entwicklung und Bedeutung der Jazzmusik, Frankfurt a. M.: Fischer 1953.

2 Zitiert nach Vivian Perlis und Libby Van Cleve, Composers' Voices from Ives to Ellington, New Haven, London: Yale University Press 2005, S. XVIII. 
Schichten blieben unter sich. Die Orte, an denen Jazz gespielt oder gehört wurde, wurden mit Misstrauen betrachtet. Kein Wunder: Hier traf man gelegentlich auf den Basler Untergrund, auf Originale, die von den „,normalen“ Bürgern gemieden wurden.

In der Schweiz erschien in diesem Jahr eine Einfübrung in die Jazæmusik, ein Büchlein des Musikwissenschaftlers Jan Sypniewski, alias Jan Slawe. Es ist das erste Jazzbuch der Schweiz, zugleich eine der ersten ernst zu nehmenden Publikationen zum Jazz in deutscher Sprache. Die Lektüre lohnt sich, denn hier ist das damalige Wissen (und Nichtwissen) zum Jazz sehr gut zusammengefasst. Einige Kernaussagen - etwas gekürzt:

„Der Jazz ist kein wissenschaftlich anerkanntes Gebiet der Tonkunst. [...] erwähnt sei noch der Umstand, dass man Jazz nur improvisieren, nicht aber nach Noten spielen kann [...]. Der Jazz wird sich also so lange von der klassischen Musik wesentlich unterscheiden, bis man ihn, entprimitivisiert' (wonach er kein Jazz mehr sein wird) [...]. Ein Jazzkunstwerk ist keine Konzeption, sondern eine spontane musikalische Äußerung der Persönlichkeit. [...] In der Jazzmusik wird ausschließlich binäre Zeitgliederung verwendet; ungerade Taktarten - wie der Dreivierteltakt - sind hier völlig unbekannt. [...] a) Das adäquate Erleben eines Jazzkunstwerkes setzt beim Zuhörer die gleiche Spontaneität voraus, die den Schöpfer im Augenblick der Schaffung desselben kennzeichnet; streng genommen, sind also nur die Neger dieses Erlebens fähig. b) Das adäquate Erleben der Jazzmusik postuliert also: [...] Ausschaltung des Intellekts [...] Reflexartige sensomotorische Reaktionsfähigkeit [...].

Nachdem nämlich die Weißen die Initiative ergriffen haben, glauben sie (oder geben vor, zu glauben), daß auch ihre Produkte ,Jazzwerke‘ sind. [...] Auf diese Weise vollzieht sich allmählich die Entprimitivisierung einer Kunst, deren ganze (außerhalb der Musik liegende) Größe und Bedeutung eben auf spontaner und primitiver Natürlichkeit basiert." ${ }^{\text {3 }}$

Es hatte sich in den Köpfen festgesetzt, dass nur schwarze Musiker genuinen Jazz spielen würden, dass weiße Musiker nur Nachahmer seien, die nie den Ausdrucksreichtum der Schwarzen erreichen könnten. Man stellte sich vor, dass mehr oder weniger glückliche Sklaven der Baumwollplantagen ohne jedes Wissen über musikalische Grundlagen ihre Musik völlig neu erfunden hätten. Viele Musiker trugen gerne zur Mythenbildung bei: die Original Dixieland Jazz Band, Errol Garner, Jelly Roll Morton etc., die ihre Vorbildung verbargen, um glaubwürdiger zu wirken. Man weiß heute, dass viele der frühen Jazzmusiker zumindest eine musikalische Grundausbildung besaßen, klassische Werke und Opern gehört, Unterrichtsliteratur durchgearbeitet hatten. Aber noch in den 1960er-Jahren habe ich junge Musiker erlebt, die jegliche musikalische Ausbildung ablehnten mit der Begründung, damit ihre Kreativität zu zerstören.

Es war für einen jungen Jazzliebhaber nicht einfach, sich eine unabhängige Meinung zum zeitgenössischen Jazzgeschehen zu bilden. Schallplatten waren teuer, spezialisierte Radiosendungen selten. Für die meisten Leute war Jazz gleichbedeutend mit Swing: Glenn Miller, allenfalls Benny Goodman. Die vom Jazz beeinflussten Schweizer Tanzorchester orientierten sich fast ausschließlich an diesem Musikstil.

3 Jan Slawe, Einführung in die Jazzmusik, Basel: Verlag National-Zeitung 1948, S. 5, 7, 13, 17, 19, 21, 49, 52. 
Nach den Don-Redman-Konzerten mit Don Byas 1946 versuchten einige Schweizer Musiker, Bebop zu spielen (Francis Burger, Jacky Seidenfuss, Hazy Osterwald) - die meisten ließen es bald wieder sein, außer dem Tessiner Flavio Ambrosetti, der wohl als Erster das Wesentliche verstand. Ihm folgte bald der Basler Pianist George Gruntz.

\section{Momentaufnahme 2: 1955, Basel}

1955 bestand das Amateur Jazz Festival Zürich bereits seit fünf Jahren. Es war eine Institution, die für den Schweizer Jazz mindestens 10 Jahre lang absolut entscheidend war. Sie führte vor allem zu einer Vernetzung der Musiker über alle Kantonsgrenzen hinweg.

Die Aufnahmen der damaligen Konzerte zeigen, dass viele Musiker noch auf einem bescheidenen technischen Level spielten. Das war nicht nur in der Schweiz so: Auch an deutschen Jazzfestivals war das Niveau kaum höher, und so ist es nicht verwunderlich, dass einzelne Schweizer Musiker an deutschen Festivals Preise gewannen.

Das Jazzbuch von Berendt verwendete 1953 zwei Seiten seines Vorworts zur Abgrenzung gegenüber „Pseudojazz“, eine Meinung, die von Musikern und Jazzfans geteilt wurde:

„Für das ungeschulte Ohr ist die Ähnlichkeit zwischen dem Jazz und den Tagesschlagern der Tanzmusik so groß, daß beide immer wieder miteinander verwechselt werden. Das ist das Hauptmißverständnis, dem die Jazzmusik seit ihrer Entstehung ununterbrochen buchstäblich ,zum Opfer fälltt. [...] Unsere Radiogesellschaften erhalten fast täglich Hörerbriefe, in denen ihnen geschrieben wird, daß sie doch nicht ,immerzu von früh bis spät Jazzmusik spielen` sollen. Wenn dann die Programmdirektoren und ihre Abteilungsleiter bestürzt in ihre Programmpläne schauen, bemerken sie, daß wirklich keine Jazzmusik, von früh bis spät ${ }^{`}$ gespielt wurde; Jazzmusik bringen die Sender allenfalls einmal in der Woche 30 oder 45 Minuten lang - und dann gewöhnlich zu später Abendstunde.

So basieren die meisten der vielen Mißverständnisse, die über die Jazzmusik im Umlauf sind, auf der Verwechslung des Jazz mit der Musik des Schlagergeschäftes. Der Jazz unterscheidet sich jedoch von der gängigen Schlager- und Tanzmusik wie in der traditionellen europäischen Musik etwa die ,Kaffeehaus'-Musik von der Sinfonik. [...]

Die Manager und Musiker des Schlagergeschäftes haben vom Jazz einen uferlosen Gebrauch gemacht. Vielleicht muß man nichts mehr an der Jazzmusik bewundern als dies: daß sie nun schon mehr als eine Generation lang einer ständigen Abnutzung und Banalisierung widerstanden hat und heute so echt und ursprünglich ist wie in den Tagen ihrer Entstehung. [...]

Es gibt kaum ein Element, kaum einen Stil, kaum eine Spielweise innerhalb der Jazzmusik, die nicht auf diese Weise geschäftlich ausgenutzt wurde. Und geschäftlich ausgenutzt, das heißt immer: verwässert und banalisiert und des ausdrucksmäßigen Gehaltes beraubt. ${ }^{4}$

Wir Musiker des Modern Jazz verstanden uns als eine Elite, die nichts zu tun hatte mit Volksmusik, mit Schlager, mit Unterhaltung. Wir trugen die Bezeichnung „Amateur“ wie eine Fahne vor uns her: Wir sahen uns als unabhängig von allen kommerziellen Überlegungen, wir wollten uns nicht bei populären Trends anbiedern. Sich zur Schau stellen auf der Bühne war

4 Berendt, Das Jazzbuch, S. 9-11. 
verpönt, die Anbiederungsgesten eines Louis Armstrong waren nicht erlaubt. Schon Mätzchen wie das elegante Antippen des Abschlusstons durch Nat King Cole waren anrüchig, Lionel Hampton mit seinen Blues-Sängern ein Verräter am wahren Jazz.

Allerdings: Jazz war um 1955 nicht einfach die Musik der „Jugend“ - dies wäre eine unzulässige Vereinfachung. Es war allenfalls eine Untergruppe der Jugend, die Jazz liebte. Gemäß einer Umfrage bestand diese weitgehend städtische Minderheit vor allem aus Studenten, Mittelschülern, der neuen Berufsgruppe der Grafiker und nur zu einem kleinen Teil (20\%) aus Handwerkern und Arbeitern. ${ }^{5}$

Bis Mitte der 1960er-Jahre sollte sich an dieser gesellschaftlichen Verankerung des Jazz wenig ändern, wie Rosenmayr, Köckeis und Kreutz in ihrer in Österreich durchgeführten Studie feststellten:

„Wegen ungenauer Fassung des Begriffs wird vielfach angenommen, daß der Jazz ,die heutige Volksmusik' darstelle; davon kann, wie Untersuchungen, einschließlich der unseren, zeigen, keine Rede sein. Bei den von uns erfaßten Jugendlichen nannten von den Schülern $10 \%$ einen Jazztitel als ihre Lieblingsplatte, von den Lehrlingen nur $1 \%$. Es wurden nur drei, durch Massenmedien besonders popularisierte ,Interpreten` von den Jugendlichen angeführt: Louis Armstrong, Benny Goodman und Chris Barber. Letzterer ist dabei nur mit einer bestimmten Schallplatte vertreten, die ,Petite Fleur' und ,Wild Cat Blues` enthält; beides Musikstücke, die zu ,Schlagern` wurden. Zu wesentlich gleichen Ergebnissen kamen G. Teindas und Yann Thireau bei französischen Jugendlichen; auch hier standen die popularisierten Interpreten Louis Armstrong und Sydney Bechet (der übrigens die Musik zu Chris Barbers Erfolgsstück, Petite Fleur' schrieb) im Vordergrund.

Ähnlich wie bei der Symphonie, Kammer- oder Klaviermusik konnten wir auch beim Jazz das Fehlen einer Beziehung zu den modernen Stilrichtungen bei den Jugendlichen feststellen. ,Modern Jazz' nannten nur insgesamt fünf höhere Schüler. Es läßt sich also auch hier die Konzentration des Interesses auf die gängigsten Stücke feststellen, die bereits mit einem Fuß den Jazz verlassen haben." ${ }^{\text {"6 }}$

Immerhin: das alljährliche Zürcher Jazzfestival vermochte den Saal des Kinos Urban (später Corso) bis in die 1970er-Jahre zu füllen, die Konzerte von Jazz at the Philharmonic, Count Basie, Duke Ellington etc. waren kommerziell selbsttragend und die Zeitungen berichteten über fast alle Konzerte, selbst von Amateurorchestern.

1952 hatte George Gruntz an einer Basler „Nuit de Jazz“ ein Oktett vorgestellt, das den Klang von Miles Davis' „Birth of the Cool“-Orchester zum Vorbild hatte. Es existiert davon nur noch die schlechte Aufnahme einer Probe und es ist darum heute fast nicht nachvollziehbar, dass dieser Auftritt die Sicht einiger junger Musiker auf den Jazz nachhaltig veränderte. Das Thema eines seiner Arrangements: das bekannte Lied La haut sur la montagne.

5 Bruno Spoerri, Untersuchungen an Jazz-Amateur-Musikern [Diplomarbeit], Zürich: Institut für Angewandte Psychologie, 1958.

6 Leopold Rosenmayr, Eva Köckeis, Henrik Kreutz, Kulturelle Interessen von Jugendlichen, Wien: Hollinek, München: Juventa 1966, S. 204 f. 
1954 erregte unser New Cool Team Aufsehen mit einer Bearbeitung des Basler Fasnachtsmarsches Morgestraich (Morgenstreich). Ein Kolumnist meinte dazu, das sei wie Mehlsuppe mit Coca-Cola, wobei Mehlsuppe die heiligste Basler Tradition verkörpert, Coca-Cola für die Unkultur der amerikanischen Barbaren steht.

Eigenkompositionen waren im Schweizer Jazz der 1950er-Jahre seltene Ausnahmen; das übliche Repertoire bestand aus den Titeln des American Songbook und der jeweils beliebtesten amerikanischen Bands.

Die damaligen Diskussionen um die Frage, was denn unter Jazz zu verstehen sei, sind heute kaum mehr vorstellbar. Der Begriff wurde äußerst eng ausgelegt: Andere als traditionelle Orchesterbesetzungen, d. h. Rhythmus und Solisten, waren undenkbar, ebenso undenkbar das Abweichen von der traditionellen Form Thema - Solo - Thema. Der Gruppendruck war groß: Sobald ein Musiker etwas Ungewohntes versuchte, hatte er Angst, es könnte nicht mehr „echter“ Jazz sein - „Pseudojazz“, „,kommerzielle Musik“ waren vernichtend ausschließende Urteile.

Noch 1955 musste in Zeitungsartikeln dem allgemeinen Publikum das Wesen des Jazz erklärt werden. Der Titel eines Textes von Jan Slawe 1960 in einem Ex Libris-Heft spricht für sich: „Hilfe! - meine Kinder sind jazzverrückt“".

Nach der vereinfachenden Jazzgeschichtsschreibung zerfiel der Jazz in wenige Stilrichtungen, die sich in einer logischen Abfolge entwickelt hatten. Wiederum: bei genauerem Hinsehen war dann alles doch nicht so glasklar - wohin gehörten Thelonious Monk? Miles Davis? Für Verfechter des alten Stils waren die neuen Tendenzen ab 1940 nicht mehr echter Jazz, für die moderneren Vertreter der sogenannten „Hot Clubs“ war die Musik der Westküste (etwa jene von Shelly Manne oder Dave Brubeck) eine unzulässige Europäisierung der ursprünglichen Musik. Aber es bahnte sich auch eine Umwälzung an, die die Jazzfans beunruhigte:

\begin{abstract}
„Das ,Rock'n'Roll'-Fieber hat mit Jazzfreunden, Jazzamateuren und Schallplattensammlern nicht das geringste zu tun. Es ist eine Mode, eine - nicht nur musikalische - Zeiterscheinung, welcher der einigermaßen vernünftig denkende, weder engstirnige noch allzu großzügige ,Normalbürger ${ }^{`}$ mit Würde und mit Gelassenheit, mit etwas Ironie und ja nicht etwa mit tierischem Ernst entgegentreten sollte." 8
\end{abstract}

\title{
Momentaufnahme 3: 1962, Zürich
}

Jazz war zu Beginn der 1960er-Jahre noch immer weit entfernt davon, in der Öffentlichkeit als Kunstform anerkannt zu werden. Jazzmusiker und -fans wurden mit Krakeelern, sog. „Halbstarken“ gleichgesetzt. Als in Zürich ein Jazzkeller projektiert wurde, gingen die Wogen hoch:

„Es wird nicht verstanden, daß mit dem Wirtschaftsbetrieb der Halbstarken bis nachts 12 Uhr eine neue Lärmquelle behördlich geschaffen wird [...]. Es soll vorgesehen sein, daß die Halbstarken den ganzen Betrieb auf eigene Kosten führen und leiten. [...] Mehr als kurios erscheint es auch, daß der

7 Jan Slawe, „Hilfe! - meine Kinder sind jazzverrückt“, in: Ex Libris 15, Nr. 1 (Januar 1960), S. 21-22; Nr. 2 (Februar 1960), S. 21-22; Nr. 3 (März 1960), S. 20-22.

8 sl. [Jan Slawe], „Rock 'n' Roll - made in Zurich“, in: Neue Zürcher Zeitung, 10. November 1956, Morgenausgabe, Blatt 7. 
gesunden Jugendbewegung wie den Pfadfindern die Benützung des Bunkers für die Durchführung gelegentlicher Führerkurse ohne Jazz und Zigarettenrauch aus grundsätzlichen Erwägungen verweigert worden sein soll. Das vorgesehene Projekt eines Jazzkellers kommt einer Kapitulation vor den Halbstarken gleich.“"

Der Jazzkritiker Jan Slawe schrieb eine Entgegnung:

„Jazzfreunde hat man in bestimmten Kreisen und in allen Ländern der Welt (bis vor wenigen Jahren auch in Staaten der kommunistischen Welt) schon immer als ,Radaubrüder', als Halbwüchsige und als obskure Elemente tituliert, und man sprach auch davon, daß die Jazzliebhaberei eine besondere Form der ästhetischen Unreife und der Primitivität der jungen Generation sei. Den Jazz betrachtete man allgemein (und man betrachtet ihn anscheinend heute noch so) als eine ausgesprochen lärmige, ja als eine nur lärmige Angelegenheit, ohne jeglichen musikalischen, ästhetischen und allgemein kulturellen Wert." $" 10$

Das alkoholfreie Cafe Africana war Anfang der 1960er-Jahre der Mittelpunkt, das „Mintons Playhouse Zürichs“ mit dem künstlerischen Leiter Remo Rau. Rau war eine Schlüsselperson im Zürcher Jazz: Er organisierte unermüdlich Konzerte, war offen für neue Ideen, gab sein Wissen auch gerne weiter.

Im Africana spielten jeweils 17-19 Uhr und 21-23 Uhr ein professioneller Pianist oder eine kleine Gruppe; die Zeit von 19 bis 21 Uhr war den sogenannten Amateuren vorbehalten. Es spielten auch Dixielandbands, von diesen ist heute jedoch kaum mehr die Rede. Die Innovation fand an den Abenden statt, wo vor allem die Gruppen um Remo Rau und Hans Kennel spielten. Hardbop (Horace Silver, Art Blakey etc.) war die Leitlinie, dazu kamen dann die Kompositionen des frühen Ornette Coleman. Das Trio von Dollar Brand (ab 1962 im Africana tätig) war für viele junge Musiker sehr wichtig, ebenso dann später die Gruppe um Chris McGregor.

Der Jazzkritiker Peter Rüedi erinnert sich:

„Jazz hatte eine Funktion. Die Musik dahinter entdeckte ich bald, aber zunächst die, die ins Schema meines juvenilen elitären Dünkels passte: den Bebop eben, die komplexeren Spielformen des Cool Jazz, dann auch den Hard Bop, obschon uns der schon fast zu eingängig war. [...] Alten Jazz verachteten wir, wie bald Rock und Pop. Zwischen den Second-Hand-Versionen des epigonalen Dixieland-Revivals und den authentischen Meistern, die eben in jener Sternstunde des Jazz Ende der Fünfziger-, Anfang der Sechzigerjahre auch ihre Renaissance erlebten, wussten wir kaum zu unterschieden [sic]. Eine erste Ahnung von authentischem altem Jazz streifte mich, als ich während einer Reise nach Wien in der dortigen Stadthalle zufällig den brachialen Kid Ory mit dem hinreissenden Henry Red Allen hörte. Aber eigentlich fiel der Groschen erst, als ich in der Zürcher ,Casa Bar` Buck Clayton begegnete, mit einer erstklassigen Schweizer Rhythmusgruppe, von der ich mich

9 sfb., „Auf krummen Wegen zum ,Jazzbunker‘ an den Unteren Zäunen“, in: Neue Zürcher Zeitung, 30. Juli 1962, Abendausgabe, Blatt 3.

10 sl. [Jan Slawe], „Von Bunkern, ,Halbstarken“ und vom Jazz, in: Neue Zürcher Zeitung, 13. August 1962, Morgenausgabe, Blatt 5. 
nur noch an den Pianisten André Hager und den Bassisten Isla Eckinger erinnere. Die ,Casa Bar war für uns vernagelte Modernisten ansonsten Feindesland, unser Revier war das ,Africana“."“11

Einige wenige Musiker machten erste Schritte in Richtung Free Jazz. Nur selten wurden sie ernst genommen.

Ein Rundschreiben der Jury an die Teilnehmer des Amateur Jazz Festival Zürich 1965:

„Der Standard des schweizerischen Amateur-Jazz gilt als einer der höchsten Europas. Beim Vergleich mit dem an ausländischen analogen Festivals Gebotenen, fällt uns jedoch in den letzten drei Jahren auf, dass diese Spitzenposition ernstlich bedroht ist. Wir machten es uns zu einer dringenden Aufgabe die Gründe, die bei uns zu einer Stagnation der Entwicklung geführt haben, aufzudecken und geben Ihnen die Ergebnisse hiermit resümiert bekannt:

Wie Sie wissen[,] bedeutet die Entfaltung der stilistischen Persönlichkeit ein Hauptelement des Jazz. Die Kopie von bereits bestehendem ist dem Jazz absolut artfremd.

Fortgeschrittene: Komponieren Sie, arrangieren Sie, versuchen Sie Ihrer Band einen persönlichen ,sound' abzugewinnen (in der Art der Instrumente, die Sie verwenden und wie Sie dieselben setzen) und versuchen Sie, Ihre solistischen Fähigkeiten mit interessanten eigenen Ideen zu bereichern. Denken Sie an die Dynamik!

Anfänger: Hände weg vom ,free jazz` / ,new thing!! Die durch diesen neusten Trend erhaltenen Freiheiten wollen ausgenützt sein, sonst wird alles sinnlose ,Falschspielerei‘. Wenn schon, dann muss diese Stilrichtung dem bereits geschulten Musiker überlassen bleiben. Wählen Sie einfache Themen und Tempi, auf welchen Sie eine saubere, swingende Musik bieten können. Die Kopie ist bei Thnen noch in dem Sinne toleriert, als dass wir wissen, dass der Anfänger am meisten durch Anhören von gutem Jazz lernen kann.

Alle: Passen Sie in jedem Fall Ihr gestecktes Ziel Ihren Fähigkeiten zum vollbringen einer sauberen Leistung• an. Alles ,so-tun-als-ob“ (zu Deutsch: ,bluff!) wird streng verurteilt. Lassen Sie sich vor dem Auftritt nicht aus der Ruhe bringen und stimmen Sie Ihr Instrument.

Es bleibt zu hoffen, dass diese wenigen, gutgemeinten Ratschläge befolgt werden, sodass wir wiederum uneingeschränkt stolz sein dürfen auf die Auslage in unserem Schaufenster: dem Internationalen Amateur Jazz Festival Zürich!

Mit besten Grüssen die Jury“"12

\section{Momentaufnahme 4: 1968}

Die Öffnung des Jazz ist in vollem Gang, aber dennoch gibt es immer noch (oder wieder) heiße Diskussionen darum, was noch als Jazz eingestuft werden darf.

Free Jazz ist einigermaßen akzeptiert, auch ideologisch verbunden mit der 68er-Bewegung; es zeigt sich auch der Beginn einer Politisierung der Musikszene. Allerdings: es ist eine kleine Minderheit unter den Musikern, die sich sozial oder politisch engagiert.

11 Peter Rüedi, Stolen Moments. 1522 Jazzkolumnen, Basel: Echtzeit 2013, S. 22 f.

12 Rundschreiben der Jury an die Teilnehmer des Amateur Jazz Festival Zürich 1965, Archiv Bruno Spoerri. 
Die neuen Feindbilder sind der sogenannte „Rock Jazz“ und jede Spielform mit Pop-Einflüssen: Wieder wird um „Kommerzialität“, um Reinheit des Jazz diskutiert.

1967 wird in Bern die erste permanente Jazzschule gegründet. Es gab zwar schon vorher einzelne Jazzkurse, die sich aber kaum auf professionellem Niveau bewegten. Musiker hatten allenfalls eine klassische Instrumental-Vorbildung. Spezifische Jazztechniken wurden durch Nachspielen von Jazzsoli aus Schallplatten, mit Hilfe von erfahrenen Kollegen und gelegentlich aus schwer erhältlichen und teuren Notenheften erlernt. Nur wenige Musiker wagten sich ins Ausland (nach Deutschland, Paris oder Schweden).

Es gibt einzelne Versuche, aus der engen Definition des Jazz auszubrechen: George Gruntz arbeitet mit tunesischen Musikern und macht erste Versuche, die Basler Tambourentradition des „Trommelns“ mit Jazz zu verbinden. Das Zürcher Metronome Quintet nimmt eine LP auf mit Techniken der elektronischen Musik. Das Hans-Kennel-Oktett spielt eine bulgarische Volksmusikmelodie und adaptiert ein Werk des Komponisten Filippo Azzaiolo aus dem 16. Jahrhundert.

Provozierende Auftritte von Free Jazz-Gruppen stoßen aber auf eindeutige Ablehnung:

„Gerade am Samstagkonzert [...] war eine Mischung von Jazz und Pop zu hören, die als abschreckendes Beispiel dafür genannt werden muß, wie man es nicht machen soll. [...] Namentlich zwölf Instrumentenbesitzer aus England, die sich ,Chris McGregor's Brotherhood of Breath' nennen, aber die Bezeichnung Musiker nicht verdienen, boten während einer ganzen Stunde auf der Corso-Bühne ein jämmerliches, chaotisches Gegeneinanderblasen und -lärmen, das jeglicher Beschreibung spottet. [...] Zum Davonlaufen wurde denn auch der Auftritt der Alexis Korner-Gruppe, die mit ihrem Pop-Rock kaum angefangen hatte, als die ,Brüderschaft ${ }^{`}$ der Chris-McGregors erneut die Bühne betrat und vollends zum instrumentalen Chaos mit überdimensionierten Verstärkeranlagen führte." ${ }^{\text {"13 }}$

\section{Fazit}

Diese kleinen Ausschnitte bezeugen es: Die Entwicklung des Schweizer Jazz vom Ende des Zweiten Weltkriegs bis Ende der 1960er-Jahre ist in keiner Weise geradlinig verlaufen und darum auch nicht einfach beschreibbar. Im Buch Jazz in der Schweiz ${ }^{14}$ haben meine Mitautoren und ich versucht, eine erste Übersicht zu schaffen; es wäre die Aufgabe der Jazzforschung, diese ersten Ansätze zu vertiefen und wenn nötig auch zu korrigieren. Heute sind noch wichtige Protagonisten dieser Zeit erreichbar, die detailliert Auskunft erteilen könnten; bald aber werden wichtige Quellen versiegen.

13 gt. [Walter Günthardt], „Jazz und Pop in buntem Gemisch. Abschluß des Festivals im Cinema Corso“, in: Neue Zürcher Zeitung, 18. September 1972, Morgen-/Mittagausgabe, S. 29.

14 Bruno Spoerri (Hrsg.), Jazz in der Schweiz. Geschichte und Geschichten, Zürich: Chronos 2005. 